\section{Assiduidade a programas de atividade física oferecidas por Unidades Básicas de Saúde: 0 discurso de participantes muito e pouco assíduos}

\section{Adherence to physical activity programs: characteristics of participants and very assiduous and less assiduous}

\author{
Natália Serra Lovato ${ }^{1,2}$ \\ Mathias Roberto Loch ${ }^{1,3}$ \\ Alberto Durán González ${ }^{4}$ \\ Maria Lucia da Silva Lopes ${ }^{1.5}$
}

\section{RESUMO}

O objetivo da pesquisa foi avaliar o discurso sobre alguns elementos da participação em grupos de prática de atividade física (AF) em usuários de programas oferecidos por duas Unidades Básicas de Saúde na cidade de Londrina/PR. Foi realizado estudo transversal, com abordagem qualitativa. Os dados foram coletados através de entrevista semi-estruturada. Os 105 usuários dos programas de AF foram divididos em cinco quintis, de acordo com a assiduidade. Foram entrevistados os sujeitos do quintil com maior assiduidade $(n=21)$ e do com menor assiduidade $(\mathrm{n}=21)$. Na análise das entrevistas, utilizou-se a análise categorial de conteúdos preconizada por Bardin. Em linhas gerais as falas revelaram que os sujeitos gostam de praticar AF, dentro ou fora do grupo, que os sentimentos vivenciados durantes as atividades eram positivos, que a motivação de familiares foi considerada importante fator de assiduidade ao programa, que muitos usuários observam melhorias na sua saúde com a participação no programa e que, entre os usuários com menor assiduidade, questões interpessoais, especialmente compromissos familiares, foram importantes para o não comparecimento em algumas atividades. Recomenda-se que a questão da aderência aos programas de AF seja levada em consideração no planejamento e replanejamento das ações, inclusive refletindo sobre as características dos programas ofertados (horário, local, tipo de atividades, etc).

\section{PALAVRAS-CHAVE}

Atividade motora; Sistema Único de Saúde; Atenção primária à saúde; Adesão; Exercício físico

\begin{abstract}
The aim of the research was assess the discourse about some elements of participation in practice groups of physical activity (PA) in programs users offered for two Health centers in Londrina/PR. Was realized a cross-sectional study with a qualitative approach. The data were collected through semi-structured interviews. The 105 members of the PA programs were divided into five quintis according to adherence. Were interviewed the subjects of the quintile with the bighest adherence $(n=21)$ and with lower adherence $(n=21)$. In the analyses of the interviews, we used categorical analysis of content proposed by Bardin. In general the statements revealed that subjects like physical activity, within or outside the group, that the feelings experienced during activities were positive, that the motivation of family was an important factor of adherence to the program, many users note improvements in your health with participation in the program and, among users with lower adherence, interpersonal issues, especially family commitments, were important to the non-attendance in some activities. The issue of adherence to physical activity programs is recommended to be taken into account in planning and replanning of actions, including reflecting on the characteristics of programs offered (the time at which beld sessions, location, type of activities, etc.).
\end{abstract}

\section{KEYWORDS}

Physical Motor Activity; Unified Health System; Primary Health Care, Adbesion; Exercise.
Rev Bras Ativ Fís Saúde p. 184-192 DOI

http://dx.doi.org/10.12820/rbafs.v.20n2p184

1 Programa de Residência Multiprofissional em Saúde da Família, Universidade Estadual de Londrina/PR.

2 Núcleo de Apoio a Saúde da Família, Secretaria Municipal de Saúde, Londrina/PR

3 Departamento de Educação Física, Universidade Estadual de Londrina

4 Programa de Pós-Graduação em Saúde Coletiva. Departamento de Saúde Coletiva, Universidade Estadual de Londrina

5 Secretaria Estadual de Saúde do Paraná, Doutora em Clínica Médica pela Universidade Federal do Rio de Janeiro. 


\section{INTRODUÇÃO}

A prática de atividade física tem sido indicada como um importante comportamento relacionado à saúde, inclusive por ser fator de proteção e de tratamento de algumas doenças crônico não transmissíveis ${ }^{1,2}$. No entanto, a prevalência de inatividade física no Brasil é elevada ${ }^{3-7}$, a despeito de diversas políticas públicas de promoção de atividade física terem sido fomentadas ${ }^{8-10}$.

A adesão e aderência à prática de atividade física é determinada por inúmeras outras variáveis que estão além do simples aquisição de conhecimento sobre os benefícios que a prática de atividade física pode propiciar ${ }^{11,12}$.

Diante disso, a adesão/aderência é um tema importante a ser estudo, à medida que se mostra um desafio para as políticas públicas de promoção de comportamentos saudáveis, inclusive relacionadas à atividade física. No entanto, estudos sobre esta temática e seus determinantes focam principalmente as atividades que ocorrem no contexto das academias de ginástica ${ }^{13-17}$, enquanto no contexto da saúde pública as pesquisas ainda são incipientes e geralmente limitam-se a investigar idosos ${ }^{18-20}$.

Desse modo, o presente estudo teve como objetivo avaliar o discurso de usuários sobre a participação em grupos de prática de atividade física de duas Unidades Básicas de Saúde (UBS) da cidade de Londrina/PR.

\section{MÉTODOS}

Foi realizado um estudo transversal com usuários de duas UBS do município de Londrina/PR.Os procedimentos metodológicos da investigação foram aprovados pelo Comitê de Ética em Pesquisa do Hospital Universitário da Universidade Estadual de Londrina (UEL).

A população alvo deste estudo foi constituída por 105 participantes que frequentaram os grupos de atividade física de duas UBS situadas na região sul da cidade de Londrina. A assiduidade dos participantes foi controlada mediante a aplicação de uma lista de chamada no início de cada sessão.

As sessões de exercícios eram sempre acompanhadas por um profissional de educação física, sendo realizadas duas vezes por semana durante uma hora. Durante as sessões, os principais exercícios trabalhados focavam a melhoria ou manutenção da flexibilidade, da resistência/força muscular, e do equilíbrio, além de atividades aeróbias, especialmente caminhada.

Para a formação da população alvo do estudo, somente foram considerados aqueles participantes que estiveram presentes no período de primeiro de junho de 2012 até 21 de dezembro de 2013, sendo que foi considerado desistente o participante que faltou um mês ou mais de atividade, sem retorno posterior a este período.

Para a seleção da amostra, os usuários foram divididos em cinco quintis de acordo com a assiduidade. Para a aplicação das entrevistas foram utilizados os participantes dos dois quintis extremos, formando, assim, o grupo dos muito assíduos (GMA, $\mathrm{n}=21$ ) e o grupo dos pouco assíduos (GPA, $n=21$ ), totalizando 42 participantes.

Para caracterização dos sujeitos foram levantadas algumas informações demográficas e de saúde, especificamente: idade, sexo, estado civil, escolaridade, situação de trabalho, distância do local da prática de atividade física, prática de alguma outra atividade física e autopercepção de saúde. 
Posteriormente, aplicou-se uma entrevista semi-estruturada, formulada com auxílio de três pesquisadores da área de atividade física e saúde e/ou saúde coletiva, com experiência em estudos qualitativos, composta por seis questões: 1) Antes do(a) sr(a) começar a participar das atividades do programa, qual(is) as suas experiências anteriores com a prática de atividade física? 2) $\mathrm{O}(\mathrm{a})$ senhor(a) gosta de fazer atividade física? $\mathrm{O}(\mathrm{a})$ senhor(a) gosta de ir ao grupo? $\mathrm{O}$ que sente no tempo que passa lá? 3) Quando o(a) sr(a) entrou no grupo, teve incentivo de alguém para começar? 4) Para continuar no grupo o(a) senhora(a) tem incentivo de alguém? 5) $\mathrm{O}$ (a) sr(a) acha que participar do grupo de atividade física ajudou a melhorar alguma coisa na sua vida ou nada mudou? Se sim, o quê? 6) A quais fatores o senhor(a) atribui suas faltas frequentes ao grupo? (esta última pergunta somente para o grupo menos assíduo).

A coleta de dados foi efetuada por uma única pesquisadora, no domicílio dos usuários selecionados, mediante prévio agendamento, nos meses de junho e julho de 2013. Em geral, as entrevistas duraram aproximadamente 30 minutos. As entrevistas foram gravadas em áudio e apagadas após a transcrição. $\mathrm{O}$ instrumento foi previamente testado em participantes dos grupos de prática de atividade física que não haviam sido selecionados para a amostra final (ou seja, não estavam nos quintis mais e menos assíduo) para que pudesse haver a familiarização da pesquisadora com o instrumento, bem como fazer algumas mudanças necessárias para a melhor compreensão das perguntas por parte dos entrevistados.

$\mathrm{Na}$ análise das entrevistas, utilizou-se a análise categorial de conteúdos preconizada por Bardin ${ }^{21}$. Para tanto, foram realizadas leituras exaustivas para o estabelecimento de categorias que pudessem melhor expressar os relatos dos participantes. As categorias utilizadas foram: gosto pela prática de atividade física, sentimentos vivenciados durante as aulas, motivação para a participação nos grupos, percepções sobre as melhorias alcançadas e motivos para falta (esta somente para o grupo menos assíduo).

\section{RESULTADOS}

\section{Características dos Participantes}

Em ambos os grupos observou-se uma elevada média de idade, baixa escolaridade, predomínio de pessoas casadas, que não tinham trabalho formal (eram aposentados ou do lar) e mais da metade referiu fazer algum tipo de atividade física fora do grupo. A proporção de mulheres foi superior no GPA (95,2\% contra 71,4\% no GMA). Em média, os usuários do GMA eram quatro anos mais velhos do que os do GPA e o tempo médio para chegar ao local aonde eram realizadas as atividades foi três minutos inferior no GMA. Todos os sujeitos entrevistados referiram ir até o grupo caminhando (Tabela 1).

\section{Discurso dos Sujeitos sobre a participação no grupo de prática de} Atividade Física

A seguir apresentam-se alguns resultados obtidos a partir das falas dos sujeitos. Para melhor organização, optou-se pela divisão em tópicos, conforme categorias definidas após a leitura das entrevistas.

- Gosto pela prática de atividade física

Em relação ao gosto pela prática de atividade física, os sujeitos de ambos os 
grupos, em geral, referiram gostar. Quatro participantes (dois de cada grupo) relataram não gostar, conforme exemplificado pelas falas abaixo.

TABELA 1 - Descrição das variáveis demográficas e de saúde dos grupos: mais assíduos (n=21) e menos assíduos (n=21).

\begin{tabular}{|c|c|c|c|c|}
\hline \multirow{2}{*}{ Variáveis Contínuas } & \multicolumn{2}{|c|}{ Muito Assíduos } & \multicolumn{2}{|c|}{ Pouco Assíduos } \\
\hline & Média & $\mathrm{DP}$ & Média & DP \\
\hline Idade & 65,9 & 11,4 & 61,8 & 11,6 \\
\hline Anos de Estudo & 4,1 & 3,4 & 4,0 & 3,2 \\
\hline $\begin{array}{l}\text { Tempo gasto para chegar ao grupo (em } \\
\text { minutos) }\end{array}$ & 6,7 & 4,0 & 9,7 & 7,5 \\
\hline \multirow{2}{*}{ Variáveis Categóricas } & \multicolumn{2}{|c|}{ Muito Assíduos } & \multicolumn{2}{|c|}{ Pouco Assíduos } \\
\hline & $n$ & $\%$ & $n$ & $\%$ \\
\hline Mulheres & 15 & 71,4 & 20 & 95,2 \\
\hline Casados (as) & 15 & 71,4 & 14 & 66,6 \\
\hline Do lar ou aposentados & 19 & 90,4 & 19 & 90,4 \\
\hline Se deslocam caminhando até o grupo & 21 & 100,0 & 21 & 100,0 \\
\hline Realizam atividade física fora do grupo & 12 & 57,1 & 11 & 52,3 \\
\hline
\end{tabular}

SMA 2: "Eu não gosto muito não, tenho preguiça. Eu vou porque se eu ficar em casa com essa idade eu irei travar".

SPA 10: "não gosto, só gosto de caminhada".

SMA 32: "Eu tenho que fazer para a saúde, obriga a gente ir, não que eu goste".

Quanto ao gostar de fazer atividade física no grupo, novamente, em geral, os participantes expressaram sentimentos positivos. Além disso, os resultados revelam sentimentos que vão para além do gosto pela prática em si, e dizem respeito à sensação de pertencimento e identidade, como exemplificado nesta fala:

SMA 4: "Gosto muito, não só pela atividade física, mas pelo fato de que o grupo fez camisetas, colocou nome no grupo, foi muito importante para mim".

Dois participantes mostraram não gostar da atividade física no grupo, sendo que um sente-se obrigado a fazer e o outro, apesar de não gostar da atividade em si, tem sentimentos positivos quanto à interação social que o grupo proporciona.

SMA 32: "Eu tenho que fazer, eu estou no grupo há muito tempo".

SPA 10: "Do grupo eu gosto de ir para conversar, mas da atividade física não, eu acho cansativa".

- Sentimentos vivenciados durante as atividades

Os participantes expressaram sentimentos positivos, muitas vezes relacionados à possibilidade de encontrar os amigos e fazer novas amizades. Outros motivos também foram mencionados com menor frequência, entre eles, a possibilidade de distrair-se dos problemas do dia a dia e de pensamentos negativos, ter um momento do dia para sair de casa, poder cuidar da saúde e de ser um apoio a outras pessoas do grupo ou de ser apoiado por elas.

SMA 2: "Você chega lá, conversa com um, conversa com outro. A atividade física afasta todos os pensamentos ruins que você tem na cabeça. Eu conbeci mais o pessoal do 
bairro, pois eu não sou muito de sair de casa".

SMA 4: "Todos que estão no grupo me apoiam e eu apoio todo mundo. Quando faço alguma coisinha errada, o meu amigo já vai lá e me ajuda, me corrige, com isso, fico muito contente também".

SMA 11: "Me sinto muito bem, porque é um borário que estou cuidando de mim. A gente conhece bastante gente, faz tanta amizade. O mais legal é que você pode incentivar aquelas pessoas que estão desanimadas, que estão pensando em parar".

Por outro lado, um participante mencionou ansiedade, especialmente em função de outros afazeres que tem que realizar na parte da manhã:

SPA 10: "Eu sinto ansiedade para vir embora, porque eu tenho muitos afazeres em casa. O horário da manhã para mim é mais difícil”.

- Motivação para a participação nos grupos

Quanto à motivação extrínseca, as falas revelam grande presença do incentivo familiar, como representados nas falas a seguir:

SMA 31: "Minha filha sempre fala para eu ir, eu chego do grupo e sempre falo para ela o que eu fiz de diferente".

SPA 38: "Minhas noras falam para eu não parar, para eu não faltar. Minhas noras não querem que eu vá à casa da minha filha cuidar da minha neta para eu não faltar". SMA 4: "Minha filha sempre liga do trabalho perguntando se eu já fui na ginástica".

Por outro lado, oito participantes (três do GMA e quatro do GPA) referiram não receber nenhum tipo de incentivo de familiares ou amigos. Porém, alguns destes demonstraram grande motivação:

SPA 16: "Não, eu mesma vou porque eu gosto muito, não preciso de incentivos".

SMA 29: "Não, eu sou o meu incentivo. Eu vou porque eu quero ter uma vida saudável, uma boa qualidade de vida, eu não tenho preguiça de ir"

- Percepção sobre melhorias alcançadas

Foi possível observar que muitos participantes, tanto do GMA como do GPA, percebiam algum tipo de melhora na saúde ao participar do grupo, como demonstram algumas falas:

SMA 3:"Melhorou. Foi muito bom, eu estava com bursite no ombro, eu não aguentava nem fazer o serviço de casa, nem pegar um copo direito, daí com o movimento melhorou, quando chegou a consulta do médico eu não sentia mais nada, fui por ir mesmo, já que a consulta tinha demorado um ano. As dores pelo corpo também melhoraram".

SMA 20: "Nossa, eu melhorei muito, eu tinha uma dor no meu cotovelo, uma dor que tinha no meu joelho também passou. Melhorou tudo no geral".

SPA 25: "Ajudou, quando eu vou no grupo, eu passo o dia maravilha, quando eu não vou, eu sinto muita falta. Minhas dores no braço também passaram, não tenho mais". SPA 37: "Melhorou, o meu braço, melhorou bem mesmo. Quando eu vou no grupo, eu durmo melhor também". 
Outros benefícios, além dos físicos, foram revelados nas falas:

SPA 9: "Consegui vencer minha timidez, sou muito tímida, não converso nem com minha vizinha aqui do lado. E lá no grupo, no começo, eu ficava toda quieta, minha irmã sempre me dizia para eu me soltar. Hoje, eu chego, já converso com todo mundo". SPA 27: "Me deu mais ânimo, disposição, distração dos problemas do dia a dia e dos afazeres de casa e fiz muitas amizades, pois quando fui para lá não conbecia ninguém". SPA 39: "Fiz muitos amigos também, antes eu morava na fazenda e não conhecia ninguém, com o grupo eu conheci muitas pessoas”.

Por outro lado, dois participantes do GPA não observaram benefícios e para um participante do GPA considerou que a melhora foi parcial.

- Motivos para faltas dos participantes pouco assíduos

Os motivos citados pelos participantes poucos assíduos para as faltas foram principalmente ligados a fatores interpessoais, como destacam algumas falas:

SPA 15: "Eu falto por causa do meu marido, porque eu tenho que cuidar dele e pelo fato de eu receber muitos parentes de fora na minha casa".

SPA 28: "Minba filha ficou grávida o ano passado e eu que tinha que levá-la de um lado pro outro".

SPA 38: "Estava cuidando dos netos. O marido da minha filha viaja muito, é caminhoneiro, quando ele viaja tenho que ir para casa da minha filba cuidar dos meus netos, pois minha filha vai trabalhar".

\section{DISCUSSÃO}

O objetivo deste estudo foi avaliar o discurso de usuários sobre a participação em grupos de prática de Atividade Física de duas Unidades Básicas de Saúde (UBS) da cidade de Londrina/PR. Em linhas gerais as falas revelaram que os sujeitos gostam de praticar atividade física (dentro ou fora do grupo), que os sentimentos vivenciados durantes as atividades eram positivos, que a motivação de familiares foi considerada importante fator de assiduidade ao programa, que muitos usuários observam melhorias na sua saúde com a participação no programa e que, entre os usuários com menor assiduidade, questões interpessoais, especialmente, compromissos familiares, foram fatores importantes para o não comparecimento em algumas atividades.

Vale mencionar que a prática de atividade física com a oferta de grupos formais de prática, é algo relativamente novo no contexto brasileiro. É possível que somente após a criação dos Núcleos de Apoio à Saúde da Família (NASF), que tem como uma de suas áreas estratégicas justamente a atividade física/ práticas corporais, é que esta oferta passou a ser mais frequente. Este aspecto é relevante inclusive por oportunizar que algumas pessoas possam praticar atividade física com orientação profissional. Por outro lado, o perfil dos usuários investigados aponta uma grande predominância de mulheres, resultado este coerente com outros trabalhos ${ }^{22,23}$. O estudo de Loch et al. ${ }^{23}$ constatou que $89,8 \%$ dos participantes de programas de atividade física de cinco UBS de Londrina eram do sexo feminino. Possivelmente, o fato de os programas 
serem oferecidos em horário comercial, inviabilize uma maior participação do sexo masculino, uma vez que a proporção de homens que trabalha fora de casa é maior à de mulheres ${ }^{24}$. Apesar de as mulheres serem a maioria nos programas, este estudo apontou que, das 21 pessoas que formaram o grupo dos muito assíduos, seis eram homens, enquanto que no grupo dos menos assíduos, somente um era homem. Ou seja, é possível que, apesar de serem minoria, aqueles homens que participam dos programas tenham uma maior assiduidade do que as mulheres. Isto pode ser reforçado se considerarmos algumas falas dos sujeitos menos assíduos sobre os motivos para as faltas. Algumas falas revelaram obrigações/compromissos familiares, tais como: cuidar do marido ou netos e ajudar a filha que estava grávida. Se culturalmente os homens trabalham mais fora de casa, estas obrigações/compromissos familiares são mais comuns entre as mulheres. De qualquer modo, maiores investigações sobre a questão da assiduidade segundo sexo, no contexto da atenção básica, seriam importantes para melhor conhecimento do tema.

Ainda sobre os dados descritivos dos sujeitos, dado interessante é que o tempo médio para chegar ao local onde eram realizadas as atividades foi três minutos inferiores no grupo dos muito assíduos. Aparentemente, três minutos pode ser um tempo muito pequeno, mas se considerarmos o percurso de ida e volta, este número dobra para seis minutos. Para pessoas com boa aptidão física e sem problemas de deslocamento este pode ser um tempo pequeno, porém para pessoas sem esta condição, esta pode ser uma variável importante para a maior ou menor assiduidade à um programa de atividade física.

O gosto pela prática de atividade física foi destacado em muitas falas. Considerando que o estudo somente incluiu usuários participantes dos programas, era esperado que o gosto pela prática estivesse presente. Em estudo com participantes de um programa de hidroginástica para idosos, o principal motivo referido pelos idosos para o ingresso no programa foi para melhorar a saúde física e mental, enquanto que os principais fatores mencionados para a permanência no programa foram o gosto pela atividade que era realizada e a sensação o gosto pela atividade que era realizada e a sensação de bem estar que a prática da atividade proporcionava ${ }^{25}$.

Os benefícios apontados pelos usuários não se limitaram à questão biológica, mas incluíam também aspectos psicológicos e sociais. Considerando que os participantes do estudo, nos dois grupos, eram constituídos em sua maioria de pessoas idosas, esses benefícios podem ser importantes para ajudar a retardar o processo de envelhecimento e fazer com que os sujeitos tenham uma vida mais ativa e autônoma, seja na família ou na comunidade.

Além dos benefícios percebidos, os sentimentos vivenciados durante a realização das atividades também evidenciam aspectos psicológicos e sociais importantes. Por outro lado, uma das falas revelou um sentimento negativo, no caso ansiedade. Esta ansiedade se dava em função das atividades físicas coincidirem com o horário dos seus afazeres domésticos que ela tinha para realizar, e complementava que o horário da manhã era "mais difícil”. De fato, é possível que, para muitas participantes destes programas a organização para participar do grupo, e também dar conta de outros afazeres, especialmente domésticos, pode não ser tarefa simples. Neste sentido, estudos futuros poderiam se ater mais especificamente a esta questão, inclusive procurando identificar se, na percepção dos usuários, o horário matutino é mesmo o melhor horário para a 
realização das atividades. Evidentemente, outros aspectos devem ser considerados na definição dos horários, como a própria organização do trabalho dos profissionais de saúde e questões climáticas.

Sobre a motivação, alguns sujeitos, mesmo referindo, não terem apoio de familiares e amigos, se mostraram motivados para a prática de atividade física. É possível que estas pessoas tenham um elevado nível de auto-eficácia, ou seja, apresentam grande confiança em sua capacidade de realizar uma ação qualquer, no caso, de praticarem atividade física. Por outro lado, alguns usuários destacaram a importância do apoio familiar, o que é coerente com as teorias/ modelos de comportamento humano que apontam que a vontade individual não é, na maioria das vezes, suficiente para que as pessoas sejam fisicamente $\operatorname{ativas}^{11,12}$.

O presente estudo teve algumas limitações, entre os quais está o delineamento transversal da pesquisa, o número de participantes, que ficou limitado aos participantes de quatro grupos de duas das $53 \mathrm{UBS}$ de Londrina/PR e a aplicação da entrevista pela própria profissional responsável pelos grupos. Porém, vale ressaltar que ainda são poucos os estudos desta natureza que os achados deste estudo podem fornecer elementos importantes para entender não só a adesão, mas também a assiduidade em grupos de atividade física no contexto das UBS.

Considerando os resultados e limitações deste estudo, recomenda-se que a questão da aderência aos programas de atividade física oferecidos pelas UBS, seja levada em consideração para o planejamento/replanejamento das ações, inclusive refletindo sobre as características dos programas ofertados (horário, local, tipo de atividades, etc). Além disso, é importante que a atuação dos profissionais envolvidos com a promoção da atividade física, ou de uma maneira mais ampla, da saúde, não seja limitada à organização de grupos específicos. Esta pode ser uma estratégia, mas não deve ser a única. Outras atividades, como, por exemplo, ações de educação em saúde, são importantes, e podem beneficiar também pessoas que não participam formalmente dos grupos.

\section{REFERÊNCIAS}

1. Kohl HW, 3rd, Craig CL, Lambert EV, Inoue S, Alkandari JR, Leetongin G, et al. The pandemic of physical inactivity: global action for public health. Lancet. 2012;380(9838):294-305.

2. Lee IM, Shiroma EJ, Lobelo F, Puska P, Blair SN, Katzmarzyk PT. Effect of physical inactivity on major non-communicable diseases worldwide: an analysis of burden of disease and life expectancy. Lancet. 2012;380(9838):219-29.

3. da Silva IC, Knuth AG, Mielke GI, Azevedo MR, Goncalves H, Hallal PC. Trends in leisure-time physical activity in a southern brazilian city: 2003-2010.J Phys Act Health. 2014;11(7):1313-7.

4. Del Duca GF, Garcia LM, da Silva SG, da Silva KS, Oliveira ES, Barros MV, et al. Clustering of Physical Inactivity in Leisure, Work, Commuting, and Household Domains: Data From 47,477 Industrial Workers in Brazil. J Phys Act Health. 2014, no prelo.

5. Hallal PC, Cordeira K, Knuth AG, Mielke GI, Victora CG. Ten-year trends in total physical activity practice in brazilian adults: 2002-2012. J Phys Act Health. 2014;11(8):1525-30.

6. Knuth AG, Malta DC, Dumith SC, Pereira CA, Morais Neto OL, Temporao JG, et al. Prática de atividade física e sedentarismo em brasileiros: resultados da Pesquisa Nacional por Amostra de Domicílios (PNAD) 2008. Cien Saude Colet. 2011;16(9):3697-705. 
7. Rech CR, Reis RS, Hino AA, Rodriguez-Anez CR, Fermino RC, Goncalves PB, et al. Neighborhood safety and physical inactivity in adults from Curitiba, Brazil. Int J Behav Nutr Phys Act. 2012;9:72.

8. Malta DC, Barbosa da Silva J. Policies to promote physical activity in Brazil. Lancet. 2012;380(9838):195-6.

9. Malta DC, Silva MM, Albuquerque GM, Lima CM, Cavalcante T, Jaime PC, et al. A implementação das prioridades da Política Nacional de Promoção da Saúde, um balanço, 2006 a 2014. Cien Saude Colet. 2014;19(11):4301-12.

10. Reis RS, Kelly CM, Parra DC, Barros M, Gomes G, Malta D, et al. Developing a research agenda for promoting physical activity in Brazil through environmental and policy change. Rev Panam Salud Publica. 2012;32(2):93-100.

11. Bauman AE, Reis RS, Sallis JF, Wells JC, Loos RJ, Martin BW. Correlates of physical activity: why are some people physically active and others not? Lancet. 2012;380(9838):258-71.

12. Sallis R, Franklin B, Joy L, Ross R, Sabgir D, Stone J. Strategies for promoting physical activity in clinical practice. Prog Cardiovasc Dis. 2015;57(4):375-86.

13. Souza JCC, Oliveira DC, Espírito-Santo GE. Percepções e motivações acerca da prática de musculação de uma academia de ginástica da baixada fluminense. Corpus et Scientia. 2012, 8(2):66-77.

14. Fermino RC, Pezzini MR, Reis RS. Motivos para a prática de atividade física e imagem corporal em frequentadores de academia. Rev. Bras. Med. Esporte. 2010, 16(1):18-23.

15. Tahara AL, Schwartz GM, Silva KA. Aderência e manutenção da prática de exercícios em academias. R. Bras. Cin. e Mov. 2003,11(4):7-12.

16. Checa FM, Furlan TE, Figueira Júnior A. Fatores determinantes para a aderência em programas de atividade física em academias de São Caetano do Sul. Revista Brasileira de Ciências da Saúde. 2006,4(10):42-4.

17. Liz CM. Motivação para a prática de musculação de aderentes e desistentes de academias [Dissertação de Mestrado]. Santa Catarina: Universidade do Estado de Santa Catarina (UDESC); 2011.

18. Nascimento MC, Silva OMP, Saggioratto ML, Vargas KCB, Schopf K, Klunk J. O desafio da adesão aos exercícios físicos em grupo de idosos em Palmitos/SC: motivos para a prática e para a desistência. Rev Bras Ativ Fís Saúde. 2010, 15(3):140-44.

19. Gomes KV, Zazá DZ. Motivos de adesão à prática de atividade física em idosas. Bras Ativ Fís Saúde. 2009,14(2):132-38.

20. Rocha SV, Carneiro LRV, Júnior LOS. Motivos para a prática de atividade física entre idosos no município de Itabuna - BA. Rev. APS. 2011, 14(3):276-82.

21. Bardin L. Análise de conteúdo. São Paulo: Edições 70, 2011.

22. Hallal PC, Tenório MCM, Tassitano RM, Reis RS, Carvalho YM. Avaliação do programa de promoção da atividade física Academia da Cidade de Recife, Pernambuco, Brasil: percepções de usuários e não-usuários. Cad. Saúde Pública. 2010, 26(1):70-78.

23. Loch MR, Rodrigues CG, Teixeira, DC. E os homens? E os que moram longe? E os que moram longe? Perfil dos usuários de programas de atividade física oferecidos pelas unidades básicas de saúde de Londrina-PR. Rev Bras Cien Esporte.2013,35(4):947-961.

24. Oliveira PR, Scorzave LG, Pazello ET. Desemprego e inatividade nas metrópoles brasileiras: as diferenças entre homens e mulheres. Nova economia. 2009, 19(2):291-324.

25. Mazo, GZ, Cardoso, FL, Aguiar, DL.Programa de hidroginástica para idosos: motivação, auto-estima e auto-imagem. Rev Bras Cin e Des Humano. 2006,8(2), p. 67-72.

\section{ENDERECO PARA}

CORRESPONDÊNCIA

MATHIAS ROBERTO LOCH.

Rua Adriano Marino Gomes, 456

bairro: Monte Belo, Londrina, PR. CEP:

86046541

E-mail: mathiasquel.br 